\title{
Mycophenolic Acid Absorption Profiles in Patients with Kidney or Combined Pancreas-Kidney Transplantation
}

\author{
Jan M. Fertmann ${ }^{1}$, Helmut P. Arbogast ${ }^{1}$, Wolf-D. Illner ${ }^{1}$, Walter Land ${ }^{1}$, \\ Karl-W. Jauch ${ }^{1}$, Johannes N. Hoffmann ${ }^{1,2 *}$ \\ ${ }^{1}$ Department of Surgery, Klinikum Grosshadern, Ludwig-Maximilians University of Munich, Munich, Germany \\ ${ }^{2}$ Department of Surgery, University of Duisburg-Essen, Duisburg and Essen, Germany \\ Email: *johannes.hoffmann@uk-essen.de
}

Received February 5, 2012; revised March 24, 2012; accepted April 5, 2012

\begin{abstract}
Background: Mycophenolate Mofetil (MMF) is widely used in organ transplant patients to avoid calcineurine inhibittor-associated side effects. Therapeutic monitoring of MMF is up to now perform by using trough level measurements (measurements before drug administration). The present study was designed to characterize potential differences in MMF absorption kinetics between patients with allogenic kidney transplantation [kidney Tx] and simultaneous pancreas kidney transplantation [PK Tx], which might for example occur due to diabetic gastrointestinal atony. Methods: A total of 64 pharmacokinetic profiles were prospectively studied in 44 adult kidney Tx and 20 PK Tx patients. To calculate AUC by the trapezoidal rule, mycophenolic acid (MPA) levels were measured in EDTA-plasma by an EMIT assay at 0, $0.5,1,2,3,4,6$, and $12 \mathrm{~h}$ after oral MMF administration between postoperative day 14 to 28 in stable patients. Results: Substantial differences between kidney Tx and PK Tx patients were evident concerning: donor age, recipient age, number of mismatches, and kidney function (serum creatinine). Despite these dissimilarities pharmacokinetic absorption profiles did not significantly differ between patient groups as measured by AUC, C2, maximum MPA concentration $\left(\mathrm{C}_{\max }\right)$, and time until maximum absorption $\left(\mathrm{T}_{\max }\right)$. Astonishingly, concomitant cyclosporine and tacrolimus medication did not influence adsorption profiles. Only MPA concentrations 6h post administration correlated closely with AUC in both patient groups, whereas trough levels failed to be predictive for AUC. Conclusions: In our study population, MMF absorption kinetics did not differ between kidney and PK Tx patients and did not seem influenced by concomitant immunosuppressive medication. Therefore, MPA measurements during the absorption phase could be useful to better estimate AUC in patients with kidney Tx and PK Tx.
\end{abstract}

Keywords: Mycophenolate Mofetil; Mycophenolic Acid; Pharmacokinetics; Kidney Transplantation; Pancreas-Kidney Transplantation

\section{Introduction}

During recent years mycophenolate mofetil (MMF), the prodrug of the immunosuppressant mycophenolic acid (MPA), has replaced azathioprine in cyclosporine A (CsA) or tacrolimus (Tac)-based immunosuppression for renal transplant patients, but also after liver, heart, and simultaneous pancreas-kidney transplantation [1,2]. The combination of cyclosporine and MMF for initial immunosuppression in renal transplant patients significantly reduced the incidence of acute rejection episodes when compared to standard therapy with cyclosporine in combination with azathioprine and methylprednisolone [3]. MMF is commonly administered in fixed doses of $3 \mathrm{~g}$ (two-times $1.5 \mathrm{~g}$ )/day or $2 \mathrm{~g}$ (two-times $1 \mathrm{~g}$ )/day without taking pharmacokinetic measurements into consideration.

\footnotetext{
"Corresponding author.
}

Recent clinical studies in renal transplant patients, however, provide some evidence that steady-state complete MMF area under curve (AUC) measurements are wellcorrelated with the likelihood of acute rejection independent from the applied MMF dosage [1]. Besides, it has been argued that transplant patients show a substantial variability in MMF absorption [4,5] partly caused by concomitant disease (e.g. renal dysfunction or liver disease) [6]. Also monitoring of inosine monophosphatase dehydrogenase (IMPDH) activity in lymphocytes as an indirect parameter of pharmacodynamic activity has been recommended [7].

Especially when changes in the immunosuppressive regimen (e.g. steroid withdrawal) are planned during the later course in stable patients, a careful evaluation of immunosuppressive MMF efforts is obligatory [7]. Besides, there is some evidence for a relationship of area 
under curve MMF concentrations and specific early absorption parameters with gastrointestinal side effects, especially during the initial absorption phase.

The present study investigates MMF absorption in non-diabetic kidney recipients and patients with simultaneous pancreas/kidney transplantation. The effect of concomitant immunosuppressive medication as well as the correlation between MMF AUC measurements and MMF absorption values was compared to investigate potential differences in MMF pharmacokinetics in these two groups which might for example be due to the gastrointestinal atony of the late diabetic syndrome.

\section{Materials and Methods}

\subsection{Patients and MPA Measurements}

A total of 64 pharmacokinetic profiles were studied in 44 adult non-diabetic kidney recipients [kidney Tx] and in 20 previously diabetic patients after simultaneous pancreas-kidney transplantation [PK Tx] from 1/2000 until 7/2002. Pharmacokinetic profiles were obtained between postoperative day 14 to 28 after reaching a clinically stable situation. A standardized daily dose of $2 \mathrm{~g} \mathrm{MMF}$ (CellCept $^{\circledR}$, Hoffmann-La Roche, Grenzach-Wyhlen, Germany) $(2 \times 1.0 \mathrm{~g})$ was given on the day of investigation as well as the day before. Pharmacokinetic profiles were evaluated while patients were still hospitalized during routine drug monitoring for standard care. Blood samples were taken immediately before oral MMF application (trough level) and $30 \mathrm{~min}, 1 \mathrm{~h}, 90 \mathrm{~min}, 2 \mathrm{~h}, 3 \mathrm{~h}$, $4 \mathrm{~h}, 6 \mathrm{~h}, 8 \mathrm{~h}$, and $12 \mathrm{~h}$ after the morning dose of MMF. EDTA-anticoagulated samples were analyzed on-line using a commercially available test system (EMIT 2000 mycophenolic acid assay, Dade Behring, Liederbach, Germany) for measurement of MPA, the active metabolite of MMF. Calibration and quality controls were performed according to the manufacturer's recommendations. Thereafter, MPA-AUC $0-12$ was calculated using the linear trapezoidal method. $\mathrm{C}_{\max }$ was taken as the largest observed MPA plasma concentration, whereas trough levels were defined as the morning predose. $\mathrm{T}_{\max }$ was defined as the time to attain $\mathrm{C}_{\max }$.

\subsection{Concomitant Immunosuppressive Medication}

In addition to MMF, 24 patients received oral cyclosporin A (Neoral ${ }^{\circledR}$, Novartis, Nuernberg, Germany), and 26 patients received oral tacrolimus (Prograf ${ }^{\circledR}$, Fujisawa, Munich, Germany). CsA-dosing was performed to achieve approximate trough levels of $150 \mathrm{ng} / \mathrm{ml}$, whereas FK 506 was applied to achieve a trough level around $10 \mathrm{ng} / \mathrm{ml}$. In the remaining 14 patients, only MMF in combination with steroids was administered. Concomitant immunosuppressive drugs were chosen according to local stan- dard protocols, e.g., addition of FK 506 for kidney recipients considered to be transplanted with an elevated immunological risk (previous transplantations with rejecttion episodes, high panel reactivity) or MMF in combination with steroids for renal transplantations in the Eurotransplant senior program. All patients received methylprednisolone in a dosage of $20 \mathrm{mg} /$ day at the time of sampling.

\subsection{Side Medication}

Among the drugs given to the patients in addition to their immunosuppressive regimen, no substances known to interfere with MPA blood levels were identified [8,9].

\subsection{Statistical Analysis}

Baseline and demographic characteristics were compared between groups by the unpaired Student t-test, or the unpaired Wilcoxon test where appropriate. Correlation coefficients were calculated by using SPSS software (SPSS version 11.0, Chicago, USA).

\section{Results}

PK Tx and kidney Tx patients did not differ with respect to gender, body weight, hematocrit, total fluid intake, total urine volume, serum albumine, and parameters of liver function (Table 1). However, patients in the PK-Tx group were significantly younger and received significantly younger transplant organs. Serum creatinine was

Table 1. Demographic parameters and organ function in patients with kidney $T x$ and pancreas-kidney $T x$.

\begin{tabular}{cccc}
\hline & Kidney Tx & Pancreas-kidney Tx & \multirow{2}{*}{ p } \\
\cline { 2 - 3 } & $(\mathrm{n}=44)$ & $(\mathrm{n}=20)$ & \\
\hline Sex (male/female) & $31 / 13$ & $13 / 7$ & NS \\
Donor age (ys.) & $48 \pm 3$ & $32 \pm 3$ & $*$ \\
Recipient age (ys.) & $50 \pm 2$ & $43 \pm 2$ & $*$ \\
Incidence of & $2 / 44$ & $20 / 20$ & $*$ \\
insulin-dependent Diabetes & & $3.8 \pm 0.2$ & $*$ \\
No of mismatches (n) & $2.6 \pm 0.2$ & $1.3 \pm 0.2$ & $*$ \\
Serum Creatinine (mg/dl) & $2.7 \pm 0.3$ & $68 \pm 3$ & NS \\
Body weight (kg) & $70 \pm 2$ & $30 \pm 2$ & NS \\
Hematocrit (\%) & $28 \pm 1$ & $3791 \pm 413$ & NS \\
Total fluid intake (ml) & $3218 \pm 225$ & $3495 \pm 388$ & NS \\
Total urine volume (ml) & $3230 \pm 277$ & $36.8 \pm 0.1$ & NS \\
Body temperature ( $\left.{ }^{\circ} \mathrm{C}\right)$ & $36.7 \pm 0.1$ & $3.5 \pm 0.2$ & NS \\
Serum albumine (g/dl) & $3.8 \pm 0.1$ & $7.5 \pm 0.9$ & NS \\
AST (U/L) & $10.5 \pm 1.4$ & $3122 \pm 240$ & NS \\
Plasma cholinesterase & $3425 \pm 207$ & & \\
(U/L) & & & \\
\hline
\end{tabular}

All values are mean \pm standard error. ${ }^{*}$ indicates $\mathrm{p}<0.05$ for patients after Kidney Tx vs. combined pancreas-kidney Tx. 
significantly lower in PK Tx patients who showed primary kidney function in $18 / 20$ patients. Despite the MMF dosage applied being identical (2 g/day), pharmacokinetic profiling showed a large interindividual variability within patients with kidney Tx and PK Tx: Using this fixed dose application regimen, MPA area under the curve $\left(\right.$ AUC $_{0-12 \mathrm{~h}}$ ) ranged from $22-144 \mu \mathrm{g} \times \mathrm{hour} / \mathrm{ml}$ and resulted in trough levels from $0.25-13.16 \mu \mathrm{g} / \mathrm{ml}$ in kidney Tx patients. Patients with PK-Tx also showed a high variability in MPA area under the curve $\left(\mathrm{AUC}_{0-12 \mathrm{~h}}\right)$ that ranged from $13-100 \mu \mathrm{g} \times$ hour $/ \mathrm{ml}$ and in trough levels $\left(\mathrm{C}_{0}\right.$ range $\left.0.49-4.95 \mu \mathrm{g} / \mathrm{ml}\right)$.

When comparing pharmacokinetic parameters obtained in kidney Tx and PK Tx patients, we could not detect any statistically significant differences (Table 2). Thus, $\mathrm{C}_{\max }$, C2 (2-h levels), C6 (6-h levels) and interestingly also $\mathrm{T}_{\max }$ (time until maximum MPA concentration is reached) did not differ between groups, subsequently resulting in comparable AUCs.

When now comparing patient groups receiving different concomitant immunosuppressive medications (cyclosporine and tacrolimus) with MMF monotherapy (Table 3), we found that kidney function was significantly improved in patients receiving a combination of MMF

Table 2. Pharmacokinetics in patients with kidney Tx and pancreas-kidney $\mathbf{T x}$.

\begin{tabular}{cccc}
\hline & Kidney Tx & Pancreas-kidney Tx & \multirow{2}{*}{ P } \\
\cline { 2 - 3 } & $(\mathrm{n}=44)$ & $(\mathrm{n}=20)$ & \\
\hline C max $(\mu \mathrm{g} / \mathrm{ml})$ & $12.4 \pm 0.8$ & $11.3 \pm 1.2$ & NS \\
C2 $(\mu \mathrm{g} / \mathrm{ml})$ & $7.3 \pm 0.7$ & $6.5 \pm 0.9$ & NS \\
T max $(\mathrm{min})$ & $77.2 \pm 7.6$ & $86.9 \pm 14.7$ & NS \\
AUC $(\mu \mathrm{g} \times$ hour $/ \mathrm{ml})$ & $54.2 \pm 3.8$ & $52.7 \pm 5.9$ & NS \\
\hline
\end{tabular}

All values are mean \pm standard error.

Table 3. Pharmacokinetics in patients with MMF monotherapy and concomitant Cyclosporine/Tacrolimus (FK 506) medication.

\begin{tabular}{|c|c|c|c|c|}
\hline & $\begin{array}{c}\text { MMF } \\
\text { monotherapy }\end{array}$ & $\begin{array}{l}\text { MMF plus } \\
\text { cyclosporine }\end{array}$ & $\begin{array}{l}\text { MMF plus } \\
\text { tacrolimus }\end{array}$ & $\mathrm{p}$ \\
\hline & $(n=14)$ & $(n=24)$ & $(n=26)$ & \\
\hline Body weight (kg) & $70.1 \pm 4.2$ & $71.9 \pm 3.1$ & $68.2 \pm 2.4$ & NS \\
\hline Creatinine (mg/dl) & $2.9 \pm 1.0$ & $2.7 \pm 0.4$ & $1.8 \pm 0.2$ & * \\
\hline Recipient Age (ys.) & $56.3 \pm 3.6$ & $46.4 \pm 3.0$ & $44.1 \pm 2.1$ & * \\
\hline $\begin{array}{c}\text { AUC } \\
(\mu \mathrm{g} \times \text { hour } / \mathrm{ml})\end{array}$ & $53.8 \pm 4.1$ & $49.1 \pm 5.2$ & $56.3 \pm 5.3$ & NS \\
\hline $\mathrm{C} \max (\mu \mathrm{g} / \mathrm{ml})$ & $12.1 \pm 1.8$ & $12.0 \pm 0.8$ & $11.9 \pm 1.2$ & NS \\
\hline $\mathrm{C} 2(\mu \mathrm{g} / \mathrm{ml})$ & $9.3 \pm 1.7$ & $7.1 \pm 0.6$ & $7.0 \pm 0.8$ & NS \\
\hline $\mathrm{T}_{\max }(\min )$ & $80.0 \pm 12.0$ & $72.9 \pm 9.8$ & $91.1 \pm 13.1$ & $* *$ \\
\hline
\end{tabular}

All values are mean \pm standard error. ${ }^{*}$ indicates $\mathrm{p}<0.05$ for MMF + Tac vs. MMF monotherapy. ${ }^{* *}$ indicates $\mathrm{p}<0.05$ for MMF + Tac and MMF + CsA vs. MMF monotherapy. plus FK 506. This observation is likely to be caused by the fact that 20/26 patients receiving this combination had undergone PK-Tx that is in general associated with shorter duration of cold ischemia, and, therefore, results in a nearly $100 \%$ primary kidney function. Combined transplantation was performed in significantly younger recipients, therefore patients receiving the combination of MMF plus FK 506 were significantly younger. Despite these intriguing differences between patient groups, we could not detect statistically significant changes in the pharmacokinetic parameters AUC, $\mathrm{C}_{\max }$ and 2 h-post dose levels (C2). Immunosuppressive comedication did only influence the time until maximum absorption $\left(\mathrm{T}_{\max }\right)$. Whereas CsA comedication reduced $\mathrm{T}_{\max }$ from $80 \mathrm{~min}$. during monotherapy to about $73 \mathrm{~min}$, combination with FK 506 even prolonged $\mathrm{T}_{\max }$ that amounted $91 \mathrm{~min}$.

Interestingly, trough levels were not found to correlate significantly with AUCs representing total drug exposure, neither in kidney transplant patients nor in PK Tx patients (Figure 1). A more close relationship was found when C2 levels were correlated with AUC (Figure 2). However, only C6 levels (6h after MMF application) showed a very close correlation with AUC, which resulted in $\mathrm{r}^{2}$-values above 0.8 (Figure 3). Interestingly, there was again no difference found between kidney Tx and PK Tx patients in absorption profiles.

\section{Discussion}

The present study was designed to compare pharma okinetics of mycophenolate mofetil (MMF) in patients after solitary kidney transplantation (Kidney Tx) withprofiles obtained in patients with simultaneous pancreas-kidney transplantation (PK Tx). Despite significant differences in donor and recipient age, occurrence of diabetes mellitus, and plasma creatinine levels, MMF was absorbed comparably in both treatment groups. Thus, there was no significant difference in maximum MMF concentrations, time until maximum absorbance, and area under curve concentration (AUC) between patients with Kidney Tx and PK Tx. This finding is in line with a recent prospective study in kidney recipients showing no existing correlation between MPA exposure and donor/recipient age as well as renal allograft function expressed as serum creatinine [10]. MMF pharmacokinetics did also not differ between patients receiving concomitant Cyclosporin A (CsA) or Tacrolimus (Tac) therapy when compared to MMF monotherapy. Interestingly, trough levels were not correlated with AUC concentrations or respective oral MMF dosage, whereas $6 \mathrm{~h}$ absorption levels after oral application showed a markedly better correlation with AUC in patients with Kidney Tx and PK Tx.

In contrast to earlier studies that report low variabilities of MMF absorption in healthy volunteers [11,12], our postoperative kidney Tx and PK Tx patients showed 


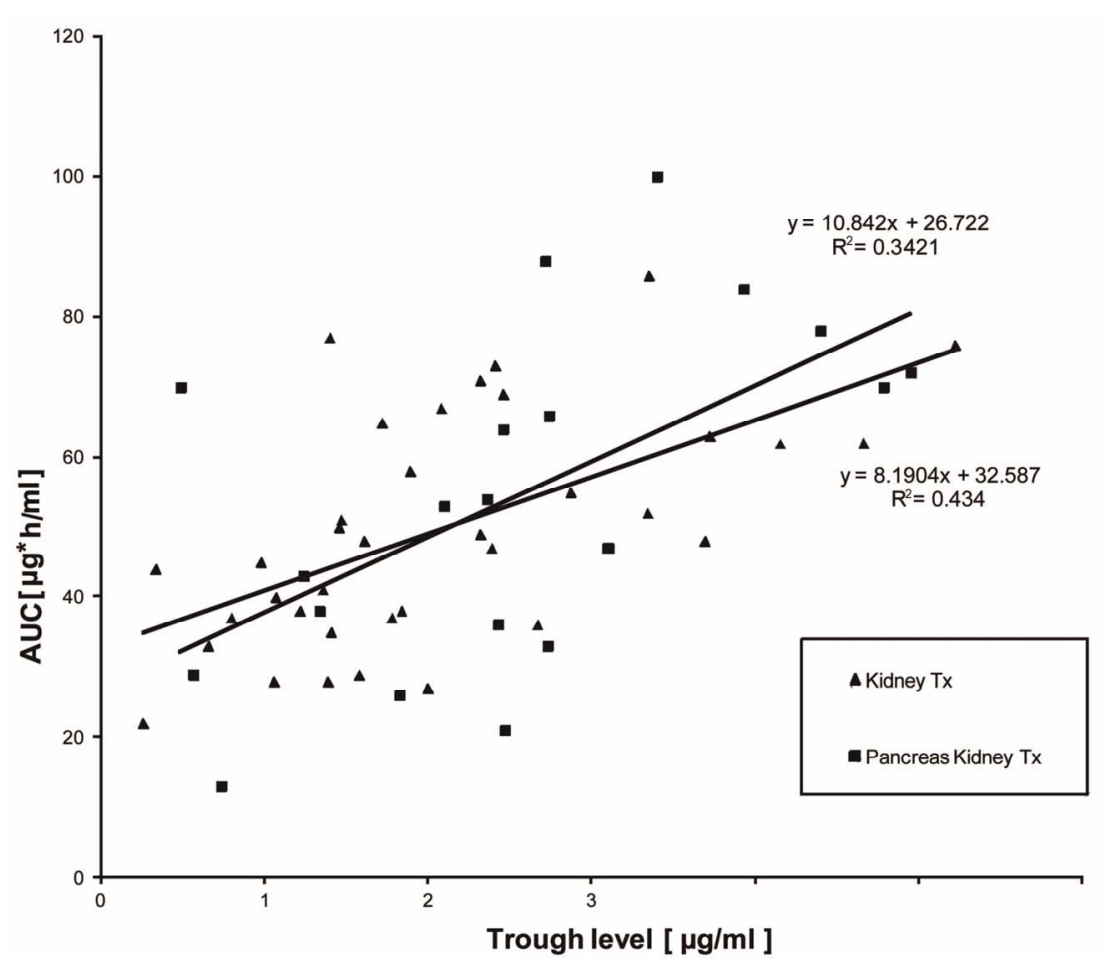

Figure 1. Correlation between trough level MPA measurements and AUC in patients with kidney Tx and pancreas-kidney Tx who were treated by a fixed dose regimen of $1 \mathrm{~g}$ MMF twice daily. Only a weak correlation was found between trough level measurements and AUC.

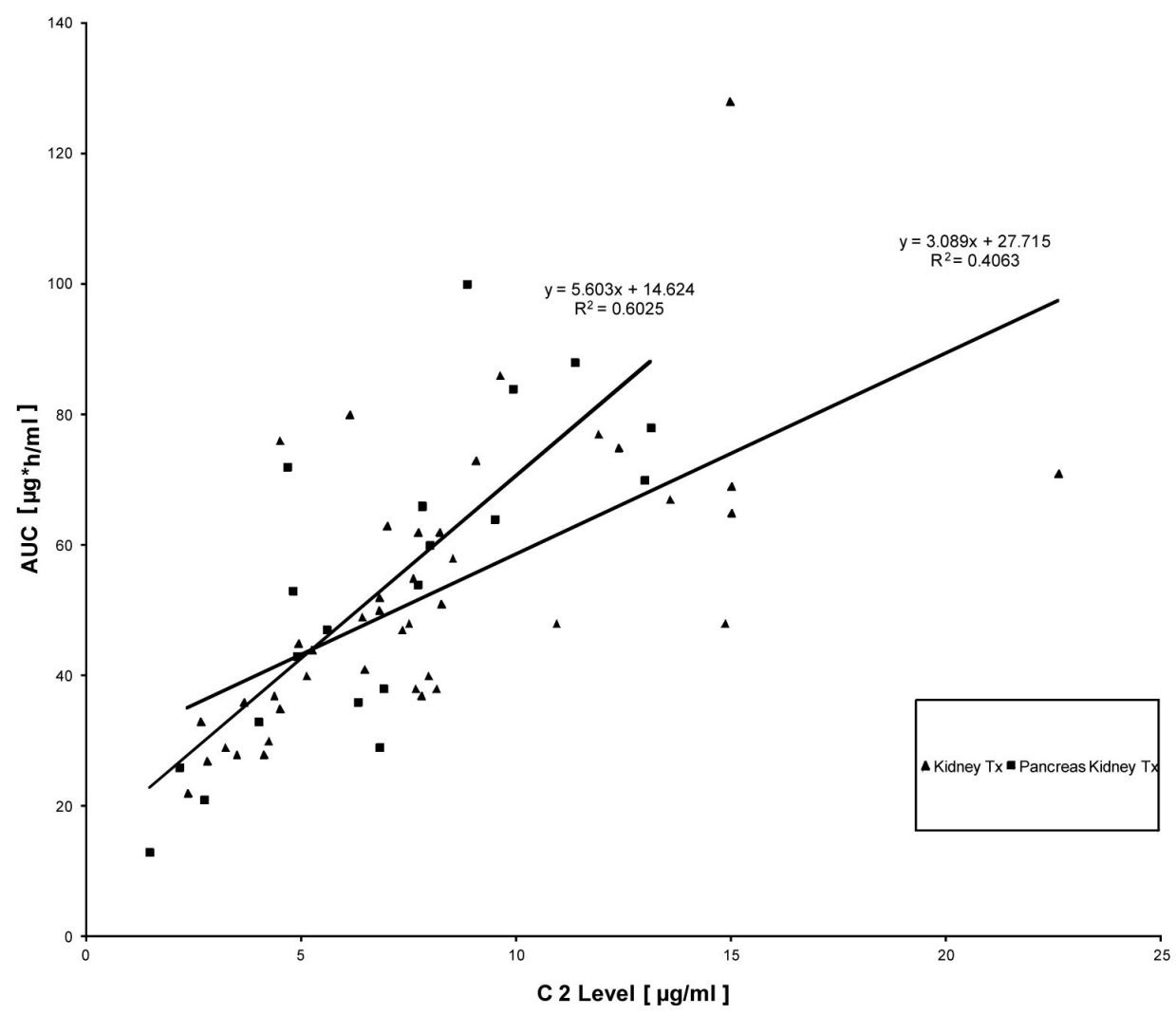

Figure 2. Correlation between 2 h post dose MPA measurements and AUC in patients with kidney Tx and pancreas-kidney Tx who were treated by a fixed dose regimen of $1 \mathrm{~g}$ MMF twice daily. 


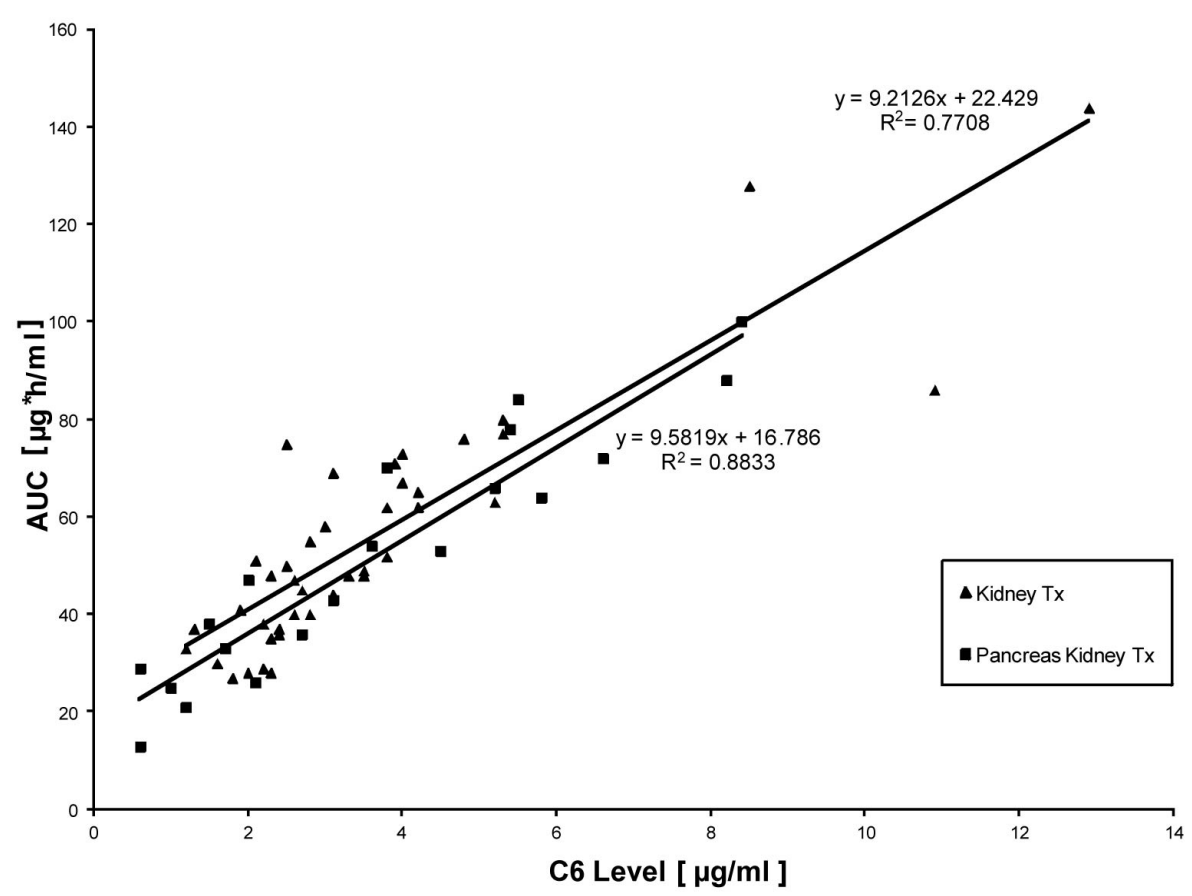

Figure 3. Correlation between $6 \mathrm{~h}$ post dose MPA measurements and AUC in patients with kidney Tx and pancreas-kidney Tx who were treated by a fixed dose regimen of $1 \mathrm{~g}$ MMF twice daily. An excellent correlation of $6 \mathrm{~h}$ post dose MPA measurements with complete AUC was found independent from the respective absolute 6 h-post dose MPA concentration.

high interindividual variabilities in MMF absorption resulting in a broad range of AUC despite the fact that all pharmacokinetic profiles were withdrawn under stable clinical conditions (no ongoing infection or rejection episode, no detectable gastrointestinal side effects) and with a sufficient time span between transplantation and the pharmacokinetic profiling (after postoperative day 14). This finding is in line with several recent reports in patients with kidney Tx who provided high interindividual variability in MMF absorption despite receiving comparable MMF dosages [1,13-15]. A similar wide range of MMF dose-adjusted MPA values has been reported for other transplant populations like heart and liver transplant patients [16]. The mechanisms of these initial differences in MMF absorption and metabolism are not yet completely understood. Furthermore, changes of MPA pharmacokinetics over time after transplantation $[6,17]$ have been demonstrated, which might be dose-dependent [10].

MMF pharmacokinetics in kidney Tx and PK Tx patients have not been directly compared, thus far, as evident from the available literature. However, it is well known that MMF absorption is highly dependent from the transplantation procedure itself, at least in some patient groups. Thus, a recent study found impressive differences in MMF pharmacokinetics when comparing renal and heart transplant recipients with a comparable daily dose of MMF [18]. Differences in MMF absorption could also be clinically significant when comparing kid- ney Tx and PK Tx patients, since the latter group could be expected to show delayed absorption when compared to kidney Tx patients. We particularly assumed differences in absorption in patients with late diabetic syndrome who are simultaneously transplanted due to postoperative gastrointestinal atony and diabetic malfunction of the gastrointestinal system, but also due to differences in the concomitant medication. Additionally, at least during the initial postoperative phase, differences in the severity of the surgical trauma that is more pronounced during PK Tx could have been related to major changes in kidney and liver function with subsequent changes in drug metabolism. Possible changes in the enterohepatic circulation that are known to be responsible for the secondary 4 - 8 hrs MMF peak, could vary significantly between groups [19]. Therefore, we decided to compare both patient groups with respect to MMF levels after oral treatment.

Interestingly, we could not detect a significant difference in MMF profiles between kidney Tx and PK Tx patients. MMF was absorbed comparably independent from age, sex and combination treatment. Concomitant FK 506 medication did not significantly change the pharmacokinetic profile when compared to Cyclosporine medication. This result was unexpected, since it is in contradiction to several reports that indicate a higher MPA exposure in renal transplant patients when given in combination with FK 506 as compared to a combination treatment with cyclosporine [20]. Also MPA trough lev- 
els were shown to be lower in combination with CsA than in kidney recipients treated with MMF and steroids alone $[21,22]$. In a study performed in rats, it was suspected that this finding was due to an inhibitory effect of CsA on bile excretion of MPA glucuronide (MPAG), to which MPA is metabolized in hepatocytes [23]. In a recent report on MMF absorption in patients after orthotopic liver transplantation, higher MPA levels were found under Tacrolimus comedication [24]. However, in the latter patients postoperatively reduced liver elimination of MMF might have been responsible for the higher trough MPA levels and even increased toxicity under the combination of MMF with FK 506. In addition to MMF, CsA or FK 506, all patients received methylprednisolone, which is also thought to influence MPA bioavailability [25]. However, the dose administered at the day of AUC sampling was identical in all groups (20 mg/day).

Measurements of trough MPA levels have been propagated to reflect at least in part drug absorption and metabolism in various transplant patients, and are currently used in transplant centers to estimate MMF drug exposure $[5,26]$. Therefore, it seemed to be interesting to investigate, whether these measurements at 12 hrs after drug exposure could in fact help to assess MMF exposure. Whereas MPA trough measurements may be applicable in the non-transplant setting [27], our results clearly show that they cannot uncritically be recommended to estimate MPA drug exposure in Kidney and KidneyPancreas transplant patients. This finding is in line with reports showing high intra- and interindividual variabilities by using this method [28]. Using other immunosuppressants, plasma concentration measurements during the absorption phase have been introduced with increasing success $[29,30]$. Thus, Cyclosporine absorption profiling and therapeutic drug monitoring using absorption measurements $2 \mathrm{~h}$ after oral Cyclosporine application has been shown to be clearly superior to trough level monitoring in kidney Tx patients [29]. It seemed, therefore, attractive to investigate whether these $2 \mathrm{~h}$-absorption measurements could also be helpful monitoring oral MMF application. We found a significantly improved correlation of 2 hrs. post dose (C2) MPA levels when compared with trough level measurements. However, only 6 h post dose (C6) MPA levels showed an excellent correlation with AUC that could be likely to be used instead of complete AUC during pharmacokinetic MMF monitoring. Especially in patients with pancreas-kidney-transplantation, a very close relationship between AUC and C6-levels was found. Since AUC is considered to be important as a predictive factor for the incidence of rejection episodes [5], this observation could indeed contribute to a simplification in MMF monitoring. Absorption levels of MPA 6h after oral application could serve as easily available surrogate parameters. Especially when compared to a limited sampling-AUC strategy $\left(\mathrm{AUC}_{0-6 \mathrm{~h}}\right)$ we found a comparable good prediction of AUC [19].

\section{Conclusion}

In conclusion, we could show comparable MMF absorption in diabetic and non-diabetic patients receiving pancreas-kidney or kidney transplantation. Combination with Tacrolimus or Cyclosporine did not change MMF pharmacokinetics. Since plasma MPA concentration 6 hrs, after application (C6) correlates better than trough level with AUC, measurement of this parameter seems useful in patients who do not allow performance of complete AUC sampling. Based on our findings, we would recommend further studies on this subject.

\section{REFERENCES}

[1] L. M. Shaw, B. Kaplan, D. DeNofrio, et al., "Pharmacokinetics and Concentration-Control Investigations of Mycophenolic Acid in Adults after Transplantation," Therapeutic Drug Monitoring, Vol. 22, No. 1, 2000, pp. 14-19. doi:10.1097/00007691-200002000-00003

[2] T. van Gelder, L. B. Hilbrands, Y. Vanrenterghem, et al., "A Randomized Double-Blind, Multicenter Plasma Concentration-Controlled Study of the Safety and Efficacy of Oral Mycophenolate Mofetil for the Prevention of Acute Rejection after Kidney Transplantation,” Transplantation, Vol. 68, No. 2, 1999, pp. 261-266. doi:10.1097/00007890-199907270-00018

[3] H. W. Sollinger, S. Cho, G. Danowitch, et al., "Мycophenolate Mofetil for the Prevention of Acute Rejection in Primary Cadaveric Renal Allograft Recipients,” Transplantation, Vol. 60, No. 3, 1995, pp. 225-232. doi:10.1097/00007890-199508000-00003

[4] G. Filler, M. Zimmering and I. Mai, "Pharmacokinetics of Mycophenolate Mofetil Are Influenced by Concomittant Immunosuppression,” Pediatric Nephrology, Vol. 14, No. 2, 2000, pp. 100-104. doi:10.1007/s004670050021

[5] M. Oellerich, M. Shipkova, E. Schütz, et al., "Pharmacokinetic and Metabolic Investigations of Mycophenolic Acid in Pediatric Patients after Renal Transplantation: Implications for Therapeutic Drug Monitoring," Therapeutic Drug Monitoring, Vol. 22, No. 4, 2000, pp. 20-26. doi:10.1097/00007691-200002000-00004

[6] L. T. Weber, T. Lamersdorf, M. Shipkova, et al., “Area under Curve Concentration-Time Curve for Total, but Not for Free, Mycophenolic Acid Increases in the Stable Phase after Enal Transplantation: A Longitudinal Study in Pediatric Patients,” Therapeutic Drug Monitoring, Vol. 21, No. 5, 1999, pp. 498-506. doi:10.1097/00007691-199910000-00002

[7] K. P. Braun, P. Glander, P. Hambach, et al., "Pharmacokinetics and Pharmacodynamics of Mycophenolate Mofetil under Oral and under Intravenous Therapy," Transplantation Proceedings, Vol. 34, No. 5, 2002, pp. 17451747. doi:10.1016/S0041-1345(02)03051-8

[8] G. Filler, N. Lepage, B. Delisle, et al., "Effect of Cyclosporine on Mycophenolic Acid Area under the Concentration-Time Curve in Pediatric Kidney Transplant Re- 
cipients,” Therapeutic Drug Monitoring, Vol. 23, No. 5, 2001, pp. 514-519.

doi:10.1097/00007691-200110000-00003

[9] C. Mignat, "Clinically Significant Drug Interactions with New Immunosuppressive Agents,” Drug Safety, Vol. 16, No. 4, 1997, pp. 267-278. doi:10.2165/00002018-199716040-00004

[10] D. Kuypers, K. Claes, P. Evenepoel, et al., "Long-Term Changes in Mycophenolic Acid Exposure in Combination with Tacrolimus and Corticosteroids Are Dose Dependent and Not Reflected by Trough Plasma Concentration: A Prospective Study in 100 De Novo Renal Allograft Recipients,” Journal of Clinical Pharmacology, Vol. 43, No. 8, 2003, pp. 866-880. doi:10.1177/0091270003256151

[11] R. E. S. Bullingham, A. Nicholls and M. D. Hale, "Pharmacokinetics of Mycophenolate Mofetil: A Short Review," Transplantation Proceedings, Vol. 28, No. 2, 1996, pp. 925-929.

[12] R. E. S. Bullingham, A. Nicholls and B. R. Kamm, "Clinical Pharmacokinetics of Mycophenolate Mofetil," Clinical Pharmacokinetics, Vol. 34, No. 6, 1998, pp. 429-455. doi:10.2165/00003088-199834060-00002

[13] L. M. Shaw, A. Nawrocki, M. Korecka, et al., "Using Established Immunosuppressant Therapy Effectively: Lessons from the Measurement of Mycophenolic Acid Plasma Concentrations,” Therapeutic Drug Monitoring, Vol. 26, No. 2, 2004, pp. 347-351. doi:10.1097/00007691-200408000-00002

[14] B. Krumme, K. Wollenberg, G. Kirste, et al., "Drug Monitoring of Mycophenolic Acid in the Early Period after Renal Transplantation,” Transplantation Proceedings, Vol. 30, No. 5, 1998, pp. 1773-1774. doi:10.1016/S0041-1345(98)00425-4

[15] Pillans PI, Rigby RJ, Kubler P, et al., “A Retrospective Analysis of Mycophenolic Acid and Cyclosporine Concentrations with Acute Rejection in Renal Transplant Recipients,” Clinical Biochemistry, Vol. 34, No. 1, 2001, pp. 77-81. doi:10.1016/S0009-9120(00)00196-X

[16] L. M. Shaw, M. Korecka, R. Venkataramanan, et al., "Mycophenolic Acid Pharmacodynamics and Pharmacokinetics Provide a Basis for Rational Monitoring Strategies,” American Journal of Transplantation, Vol. 3, No. 5, 2003, pp. 534-542. doi:10.1034/j.1600-6143.2003.00079.x

[17] V. C. Cox and M. Ensom, "Mycophenolate Mofetil for Solid Organ Transplantation: Does the Evidence Support the Need for Clinical Pharmacokinetic Monitoring?” Therapeutic Drug Monitoring, Vol. 25, No. 2, 2003, pp. 137157. doi:10.1097/00007691-200304000-00003

[18] L. M. Shaw, M. Korecka, D. DeNofrio, et al., "Pharmacokinetic, Pharmacodynamic, and Outcome Investigations as the Basis for Mycophenolic Acid Therapeutic Drug Monitoring in Renal and Heart Transplant Patients," Clinical Biochemistry, Vol. 34, No. 1, 2001, pp. 17-22. doi:10.1016/S0009-9120(00)00184-3

[19] A. G. Johnson, R. J. Rigby, P. J. Taylor, et al., "The Kinetics of Mycophenolic Acid and Its Glucuronide Metabolite in Adult Kidney Transplant Recipients," Clinical Pharmacology \& Therapeutics, Vol. 66, No. 5, 1999, pp.
492-500. doi:10.1016/S0009-9236(99)70012-3

[20] K. Zucker, A. Rosen, A. Tsaroucha, et al., "Unexpected Augmentation of Mycophenolic Acid Pharmacokinetics in Renal Transplant Patients Receiving Tacrolimus and Mycophenolate Mofetil in Combination Therapy, and Analogous in Vitro Findings,” Transplant Immunology, Vol. 5, No. 3, 1997, pp. 225-232. doi:10.1016/S0966-3274(97)80042-1

[21] P. J. Smak Gregoor, T. van Gelder, C. J. Hesse, et al., "Mycophenolic Acid Plasma Concentrations in Kidney Allograft Recipients with or without Cyclosporin: A CrossSectional Study," Nephrology Dialysis Transplantation, Vol. 14, No. 3, 1999, pp. 706-708. doi:10.1093/ndt/14.3.706

[22] L. Pou, M. Brunet, C. Cantarell, et al., "Mycophenolic Acid Plasma Concentrations: Influence of Comedication,” Therapeutic Drug Monitoring, Vol. 23, No. 1, 2000, pp. 35-38. doi:10.1097/00007691-200102000-00007

[23] T. van Gelder, J. Klupp, M. J. Barten, et al., “Comparison of the Effects of Tacrolimus and Cyclosporin on the Pharmacokinetics of Mycophenolic Acid,” Therapeutic Drug Monitoring, Vol. 23, No. 2, 2001, pp. 119-128. doi:10.1097/00007691-200104000-00005

[24] M. Glanemann, J. Klupp, J. M. Langrehr, et al., "Higher Immunosuppressive Efficacy of Mycophenolate Mofetil in Combination with FK 506 than in Combination with Cyclosporine A,” Transplantation Proceedings, Vol. 32, No. 3, 2000, pp. 522-523. doi:10.1016/S0041-1345(00)00872-1

[25] D. Cattaneo, Perico N., F. Gaspari, et al., "Glucocorticoids Interfere with Mycophenolate Mofetil Bioavailability in Kidney Transplantation," Kidney International, Vol. 62, No. 3, 2002, pp. 1060-1067. doi:10.1046/j.1523-1755.2002.00531.x

[26] G. I. Hubner, R. Eismann and W. Sziegoleit, "Relationship between Mycophenolate Mofetil Side Effects and Mycophenolic Acid Plasma Trough Levels in Renal Transplant Patients,” Arzneimittelforschung, Vol. 50, No. 10, 2000, pp. 936-940.

[27] I. Neumann, M. Haidinger, H. Jaeger, et al., "Pharmacokinetics of Mycophenolate Mofetil in Patients with Autoimmune Diseases Compared to Renal Transplant Recipients," Journal of the American Society of Nephrology, Vol. 14, No. 3, 2003, pp. 721-727. doi:10.1097/01.ASN.0000051598.12824.DA

[28] M. Brunet, J. Martorell, F. Oppenheimer, et al., "Pharmacokinetics and Pharmacodynamics of Mycophenolic Acid in Stable Renal Transplant Recipients Treated with Low Doses of Mycophenolat Mofetil," Transplant International, Vol. 13, No. S1, 2000, pp. S301-S305.

[29] P. Belitsky, G. A. Levy and A. Johnston, "Neoral Absorption Profiling: An Evolution in Effectiveness,” Transplantation Proceedings, Vol. 32, No. 3A, 2000, pp. 45S52S. doi:10.1016/S0041-1345(00)00863-0

[30] K. Mahalati, P. Belitsky, I. Sketris, et al., "Neoral Monitoring by Simplified Sparse Sampling Area under the Concentration-Time Curve,” Transplantation, Vol. 68, No. 1, 1999, pp. 55-62. doi:10.1097/00007890-199907150-00011 